\title{
Coulomb drag between one-dimensional Wigner crystal rings
}

\author{
John Baker and A G Rojo \\ Department of Physics, University of Michigan, Ann Arbor, MI 48109-1120, USA
}

Received 16 January 2001

\begin{abstract}
We consider the Coulomb drag between two metal rings in which the longrange Coulomb interaction leads to the formation of a Wigner crystal. The first ring is threaded by an Aharanov-Bohm flux, creating a persistent current $J_{0}$. The second ring is brought into close proximity to the first and due to the Coulomb interaction between the two rings a drag current $J_{\mathrm{D}}$ is produced in the second. We investigate this system at zero temperature for perfect rings as well as the effects of impurities. We show that the Wigner crystal state can in principle lead to a higher ratio of drag current to drive current $J_{\mathrm{D}} / J_{0}$ than in weakly interacting electron systems.
\end{abstract}

\section{Introduction}

Coulomb drag between current-carrying systems has been studied in numerous papers both experimentally and theoretically [1-6]. The earliest experiments were done in twodimensional systems. In this configuration the electrons are confined to two-dimensional layers. In one such layer a current is driven and another conducting layer is brought to within close proximity of the first. The Coulomb interaction between the electrons in these two layers causes a transfer of momentum and the second layer acquires a current. This current is referred to as the drag current.

Recently, the phenomenon of Coulomb drag in one-dimensional systems has attracted much attention, particularly with regard to nanowires and nanotechnology [7-11]. The basic description of the one-dimensional case is identical to that in two dimensions. In the typical setup, a current is driven in a one-dimensional wire and a second wire is brought close to the first. The Coulomb interaction between the charges in these wires causes a drag current to be produced in the second wire. In all of these systems the time-averaged charge density of the systems is translationally invariant. The coupling of the two systems is due to charge fluctuations therein.

It is known, however, that the long-range character of the Coulomb interaction can lead to the formation of a Wigner crystal in which the electrons become localized and form a periodic lattice. Numerical simulations on one-dimensional systems indicate that an arbitrarily weak long-range interaction will lead to the formation of a Wigner crystal [12, 13]. This long-range nature of the Coulomb force should be apparent for low electron densities. 
In this paper we consider the drag between two such Wigner crystals. The principle difference between our investigation and those of [7-11] is not in the particular setup (i.e. rings versus straight wires) but in the nature of the charge density of the system. It is evident that the Wigner crystal has a non-uniform charge distribution and we will show that this in principle can lead to a much higher value of the drag current.

\section{Classical drag}

It is the aim of this section to analyse from a classical perspective some aspects of current drag that we may expect in a quantum mechanical treatment of the Wigner crystal. The Wigner crystal is a system where the electrons are effectively localized, so it is reasonable to expect that some aspects of the current drag between Wigner crystals may be exhibited in a classical system.

Here, we discuss the current drag between two one-dimensional wires consisting of classical particles interacting via an unscreened Coulomb potential. The two systems are close enough that they are coupled by the Coulomb interaction between the wires. This system is illustrated in figure 1 . To get an idea of what to expect from such a system we first consider a 'toy model' consisting of two lines of equally spaced particles interacting via a periodic potential and moving under a viscous drag force. The main difference between this system and that in figure 1 is that the spacing between particles is fixed so that the internal dynamics of the system are ignored. The description of such a system has been considered by Doering [14] and in an analysis by Cladis et al [15] in the context of the vortex dynamics in type-II superconductors.

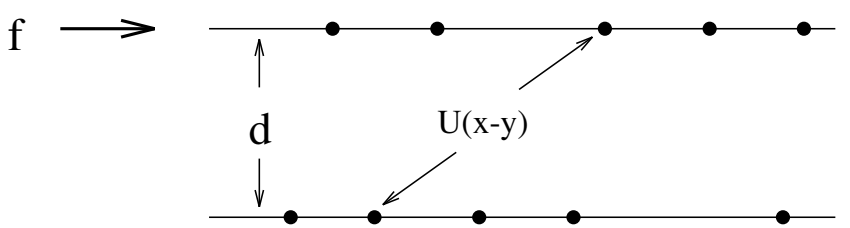

Figure 1. Illustration of the current drag setup for considered in this classical analysis. A constant force $f$ drives system one. $U(x-y)$ is the interwire interaction and $d$ is the distance of separation.

In this analysis, two rigid chains of equally spaced particles are taken in the presence of a viscous drag force $F_{\mathrm{d}}=\alpha \dot{x}$. Since each chain is rigid, its position can be denoted by the position of one of its particles. Call $x_{1}$ the position of chain 1 and $x_{2}$ the position of chain 2. The interaction between the two chains is then periodic in $x_{1}-x_{2}$ and modelled by $F_{i}=A \sin \left[(2 \pi / a)\left(x_{1}-x_{2}\right)\right] *$ where $a$ is the lattice spacing. In addition, the first chain is driven by a constant force $f$. In a steady state the equations of motion of the two chains are given by

$$
\begin{aligned}
& \alpha \dot{x_{1}}=-A \sin \left[(2 \pi / a)\left(x_{1}-x_{2}\right)\right]+f \\
& \alpha \dot{x_{2}}=A \sin \left[(2 \pi / a)\left(x_{1}-x_{2}\right)\right] .
\end{aligned}
$$

Setting $\phi=\left(x_{1}-x_{2}\right)$, results in the following equation for the difference between the velocities of the two chains:

$$
\dot{\phi}=f / \alpha-\frac{2 A}{\alpha} \sin (2 \pi \phi / a)
$$

* As pointed out in reference [15], the exact form of the interaction is not important for the results so long as the force is periodic in the vortex lattice. 
with solution

$$
T_{0}=\int_{0}^{a / 2} \frac{\mathrm{d} \phi}{\frac{f}{\alpha}-\frac{2 A}{\alpha} \sin (2 \pi \phi / a)}
$$

where $T_{0}$ is the time for the two chains to slip one complete cycle (distance $a / 2$ ).

Carrying out the integral results in

$$
T_{0}=\left\{\begin{array}{lll}
\infty & \text { for } f<2 A & \text { (locking) } \\
\frac{a}{2} \frac{1}{(f / \alpha)^{2}-(2 A / \alpha)^{2}} & \text { for } f>2 A & \text { (slipping) }
\end{array} .\right.
$$

This simple analysis shows the interesting result that, for suitable conditions of the parameters $f$ and $A$, the two chains will be locked together with the drag current equal to the drive current.

We now wish to investigate the behaviour of this classical system when internal dynamics are included. We take therefore as a model of the dynamics of these classical particles the following Fokker-Planck equation:

$$
\begin{aligned}
\frac{\partial}{\partial t} P(\vec{x}, \vec{y}, t)= & \sum_{i} D\left(\frac{\partial^{2}}{\partial x_{i}^{2}}+\frac{1}{k_{\mathrm{B}} T} \frac{\partial}{\partial x_{i}} F_{i}\right) P(\vec{x}, \vec{y}, t) \\
& +\sum_{j} D\left(\frac{\partial^{2}}{\partial y_{j}^{2}}+\frac{1}{k_{\mathrm{B}} T} \frac{\partial}{\partial y_{j}} F_{j}\right) P(\vec{x}, \vec{y}, t)
\end{aligned}
$$

Here, $\vec{x}=\left(x_{1}, x_{2} \ldots, x_{N}\right)$ labels the positions of $N$ particles in wire $1, \vec{y}=\left(y_{1}, y_{2} \ldots, y_{N}\right)$ labels the positions of particles in wire 2, $D$ is the diffusion constant and $T$ is the temperature. $P(\vec{x}, \vec{y}, t)$ is then the probability of having the $N$ particles in layer 1 at positions $\vec{x}$ and the $N$ particles in layer 2 at position $\vec{y}$ at time $t$. The forces present are

$$
F_{i}=f+\frac{\partial}{\partial x_{i}} V(\vec{x})+\frac{\partial}{\partial x_{i}} U(\vec{x}, \vec{y})
$$

in wire 1 and

$$
F_{j}=\frac{\partial}{\partial y_{j}} V(\vec{y})+\frac{\partial}{\partial y_{j}} U(\vec{x}, \vec{y})
$$

in wire 2. The interaction $U$ is taken to be the unscreened Coulomb interaction between the wires, $V$ is the unscreened Coulomb interaction within each wire and $f$ is a constant force driving the particles in wire 1 . The basic problem is to determine the drag current produced in wire 2 due to the moving charges in wire 1.

In the numerical analysis of this problem we performed Monte Carlo simulations on discrete systems. The conducting systems are partitioned into a lattice with the particles occupying positions at the lattice points. The dynamics of the simulation are determined by equation (1) which is approximated numerically as follows. For each particle, one of two possible random directions is chosen. If the particle lowers its energy by moving one lattice site in that direction, then the move is accepted. Otherwise the move is accepted with probability $\mathrm{e}^{-\beta \Delta E}$, where $\beta=1 / k_{\mathrm{B}} T$ and $\Delta E$ is the change in the particle's energy upon making this move. This process is completed iteratively for each particle until a steady-state condition is reached. Simulations were performed to determine the dependence of the drag current on separation between the two systems. The results are shown in figure 2.

The plot of drag current versus separation shows the fact that the sum of the drive and drag currents is a constant, $\bar{J}_{\mathrm{d}}+\bar{J}_{0}=\frac{N D f}{k_{\mathrm{B}} T}$, as can be seen by averaging the right-hand side of equation (1), (RHS $=\sum_{i} \frac{\partial}{\partial x_{i}} J_{0}+\sum_{j} \frac{\partial}{\partial y_{j}} J_{\mathrm{d}}$ ). It also shows that the drag current is significant only when the distance of separation is of the order of the interparticle spacing or smaller. 


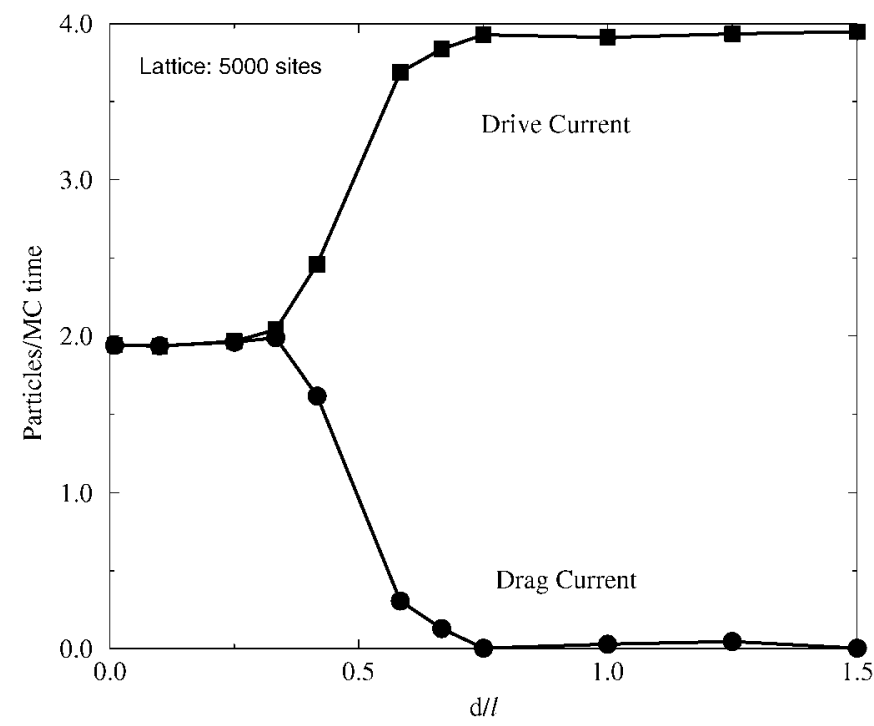

Figure 2. Plot of drag current against wire separation for two one-dimensional wires.

The main feature of interest in this plot, however, is that the drag current, $J_{\mathrm{d}}$, is essentially equal in magnitude to the drive current, $J_{0}$, at small interwire separations. As the separation between the wires is increased the drag current shows a smooth decrease in magnitude. The main difference between this plot and the toy model (where internal dynamics are ignored) is that there does not appear to be an abrupt locking transition. However at sufficiently large values of the interaction parameter, $A$, the two chains are essentially locked together with the drag current equal in magnitude to the drive current. We will now examine to what extent this classical effect is exhibited in a quantum mechanical system.

\section{Wigner crystal ring}

\subsection{Disorder-free case}

In this section we calculate the non-dissipative drag between two perfect Wigner crystal rings at zero temperature. The basics of non-dissipative drag effects have been considered in previous papers $[16,17]$. The basic system of interest here consists of two metallic rings one of which is threaded by an Ahranov-Bohm flux, $\Phi$. This flux shifts the ground state, $E_{\mathrm{g}}$, of the ring to a current-carrying state which is calculated according to

$$
J=\frac{\partial E_{\mathrm{g}}}{\partial \Phi} .
$$

If another ring is brought close to the first one, then the Coulomb interaction will cause a drag current to be produced in the second ring. Since this is the ground state of the two-ring system this current is non-dissipative and persists as long as the magnetic flux is present in the first ring. The difficulties of realizing such a geometry have been addressed in [17]. In particular, it is assumed that the magnetic flux only penetrates the first ring and the flux through ring 2 is zero. Reference [17] gives several geometries in which such a situation might be realized. In the following we will assume that the flux only penetrates ring 1 and ignore the difficulties involved with actually creating such a system. 
In prior works dealing with the nondissipative drag, the two rings have uniform charge densities and the drag effect is due to coupling of charge fluctuations in each ring. In a system with zero disorder, the relative magnitude of the drag current produced is typically a small fraction of the driving current. For two such systems separated by a distance of $\sim 200 \AA$ the drag current is on the order of $\sim 10^{-4}$ times the current in the driving system [16]. Based on the analysis of the last section, we expect that the drag current of two Wigner crystals could in principle be as large as the driving current.

The model of the one dimensional Wigner crystal that we use here is that proposed in [18] and [19]. In this model the Wigner crystal is viewed as an elastic chain of spinless electrons. In the continuum limit, the Wigner crystal of length $L$, in the presence of an Ahranov-Bohm flux, $\Phi$, is described by the Lagrangian [19]

$$
\mathcal{L}=\int \mathrm{d} x\left[\frac{m}{2 a}\left(\dot{u}^{2}-s^{2}\left(u^{\prime}\right)^{2}\right)+\frac{\hbar}{L} \frac{\Phi}{\Phi_{0}} \dot{u}\right]
$$

where $a$ is the crystal period, $m$ is the electron mass, $s$ is the velocity of sound in the crystal and $\Phi_{0}$ is the flux quantum. The field variable $u(x, t)$ describes the local displacement of the chain at a point $x$ at time $t$. If the field variable is expanded in a Fourier series

$$
u=\frac{1}{\sqrt{L}} \sum_{k} c_{k} \mathrm{e}^{\mathrm{i} k \theta}
$$

we get

$$
\mathcal{L}=(\mu / 2) \sum_{k}\left(\dot{c}_{k}^{2}-s^{2} k^{2} c_{k}^{2}\right)-\frac{\hbar}{L} \tilde{\Phi} \dot{c}_{0}
$$

where $\tilde{\Phi}=\Phi / \Phi_{0}$. It is clear that the flux will only couple to the zero mode of the ring. So in dealing with the persistent current of a disorder-free Wigner crystal ring, the internal dynamics of the ring may be ignored and only the rotation of the ring as a whole is relevant. We wish to study the interaction between two such Wigner crystal rings as shown in figure 3 . We take as

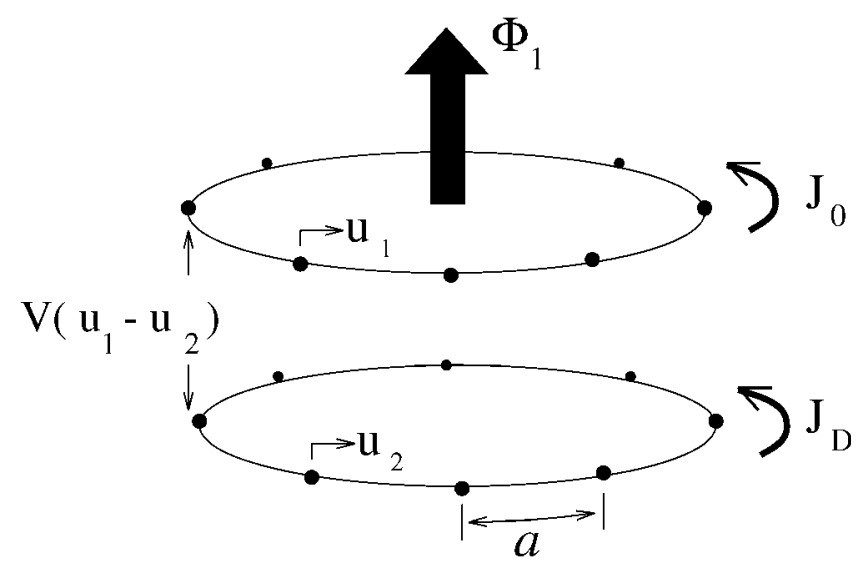

Figure 3. Schematic illustration of the two-ring Wigner crystal setup. The crystal period is $a$ and $u_{1}(x, t)$ denotes the local displacement of ring 1 and $u_{2}(x, t)$ denotes the local displacement of ring 2. Ring 1 has an Ahranov-Bohm flux, $\Phi_{1}$, present which creates a persistent current $J_{0}$ in ring 1 . The interaction between the two crystals, $V\left(u_{1}-u_{2}\right)$ is a function of the relative displacement of the two crystals and creates a drag current $J_{\mathrm{D}}$ in ring 2. 
a model for two disorder-free interacting Wigner crystal rings the following Hamiltonian

$H=\frac{1}{2 M}\left(-\mathrm{i} \hbar \frac{\partial}{\partial u_{1}}-\frac{e}{c} \Phi_{1}\right)^{2}+\frac{1}{2 M}\left(-\mathrm{i} \hbar \frac{\partial}{\partial u_{2}}-\frac{e}{c} \Phi_{2}\right)^{2}+V_{0} \cos \frac{2 \pi}{a}\left(u_{1}-u_{2}\right)$

where $M=N m$ is the total mass of each ring, $V_{0}$ represents the strength of the inter-ring interaction, $\Phi_{1}$ is the flux through ring $1, \Phi_{2}$ is the flux through ring 2 and $u_{1,2}$ represents the displacement of ring 1, 2 respectively. The first two terms in equation (3) represent the individual dynamics of rings 1 and 2 respectively. The third term represents the interaction between the two rings. This interaction term exhibits the modulations in the charge density of our system and it is evident that the interaction between the chains is periodic in the relative displacement between the two crystals. For simplicity we model this interaction as a cosine term; however, the basic features of this analysis should not depend on the particular form of the periodic potential used.

Transforming to centre of mass and relative angular coordinates $\phi=\frac{2 \pi}{L}\left(u_{1}-u_{2}\right)$ and $\theta=\frac{\pi}{L}\left(u_{1}+u_{2}\right)$, we have in dimensionless form

$$
\left[\left(\mathrm{i} \frac{\partial}{\partial \phi}+\frac{\alpha}{2}\right)^{2}+\frac{1}{4}\left(\mathrm{i} \frac{\partial}{\partial \phi}+\beta\right)^{2}+q \cos (N \phi)\right] \Psi=\epsilon \Psi
$$

where $\alpha=\Phi_{1}-\Phi_{2}, \beta=\Phi_{1}+\Phi_{2}, q=V_{0}\left(M R^{2} / \hbar^{2}\right)$ and $\epsilon=E\left(M R^{2} / \hbar^{2}\right)$. Our task is now to solve equation (4) for the eigenenergy $\epsilon$.

The Hamiltonian, equation (4), separates into two parts $H=H_{\phi}+H_{\theta}$ with energies $\epsilon=\epsilon_{\phi}+\epsilon_{\theta}$. The ground state wavefunction is a product $\Psi(\phi, \theta)=S(\theta) T(\phi)$. The equation for the centre of mass coordinates is readily solved, giving

$$
\begin{aligned}
& S(\theta)=\frac{1}{\sqrt{2 \pi}} \mathrm{e}^{\mathrm{i} n^{\prime} \theta} \\
& \epsilon_{\theta}=\frac{1}{4}\left(n^{\prime}+\beta\right)^{2} \quad n^{\prime}=0, \pm 1, \pm 2, \ldots .
\end{aligned}
$$

The equation for the relative coordinate wavefunction is

$$
\left[-\left(\mathrm{i} \frac{\partial}{\partial \phi}+\frac{\alpha}{2}\right)^{2}-q \cos (N \phi)+\epsilon_{\phi}\right] T(\phi)=0 .
$$

For $\alpha=0$ this reduces to Mathieu's equation. The energy eigenvalues correspond to the periodic solutions $T(0)=T(2 \pi)$. We therefore substitute the Fourier expansion

$$
T=\sum_{-\infty}^{\infty} c_{n} \mathrm{e}^{\mathrm{i} n \phi}
$$

into equation (6) and obtain the following recursion relation for the coefficients $c_{n}$ :

$$
\frac{q}{2} c_{n+1}+\left(\epsilon-[n-\alpha]^{2}\right) c_{n}+\frac{q}{2} c_{n-1}=0
$$

The energy eigenvalues may be found numerically to any desired accuracy by truncating the set of equations for some $c_{N}$ and searching for the value of $\epsilon$ which gives a zero value for the appropriate determinant of coefficients [20].

The energy eigenvalues determined in this way, combined with the results of equation (5), allow the currents in each ring to be determined from $J_{1}=\frac{\partial \epsilon}{\partial \Phi_{1}}$ and $J_{2}=\left.\frac{\partial \epsilon}{\partial \Phi_{2}}\right|_{\Phi_{2}=0}[17]$. The results are shown in figure 4. 

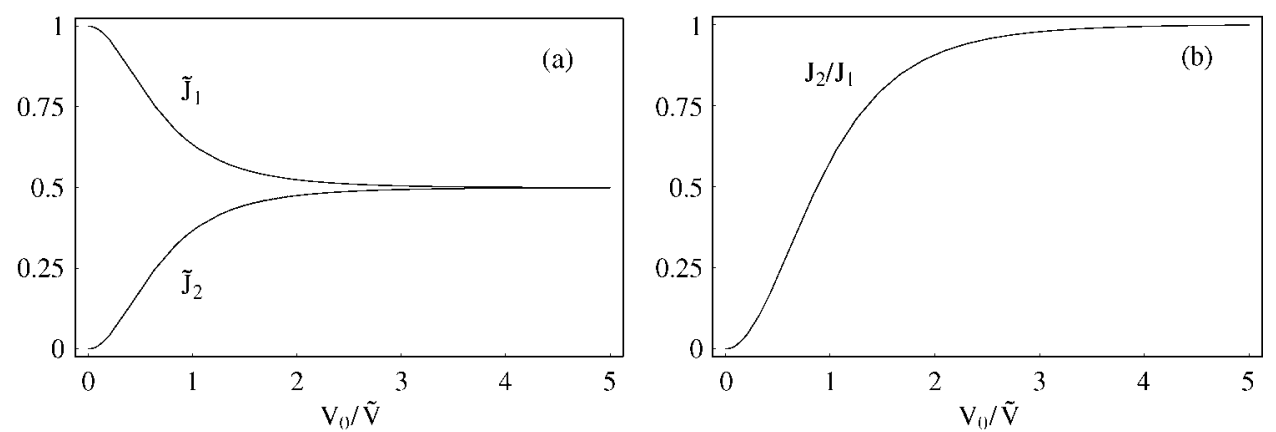

Figure 4. Plots of drive and drag current against the interaction strength. $V_{0}$ is shown in units of $\tilde{V}=\frac{N \hbar^{2}}{m R^{2}}$ and the current $\tilde{J}=J / J_{1}(0)$ where $J_{1}(0)$ denotes the current in ring 1 at $V_{0}=0$.

Figure 4 shows that the drag current does approach the drive current in magnitude as the interaction $V_{0}$ is increased which is qualitatively similar to the classical result. For large values of the interaction strength, $q / N \gtrsim 3$ or

$$
V_{0} \gtrsim 3 \hbar^{2} N / m R^{2}
$$

the two Wigner crystals have essentially the same current with each having half the current that one ring would have if isolated.

We want to relate the interaction in equation (3) to the true Coulomb interaction between our two Wigner crystals. We return to the discrete lattice picture and approximate $V_{0}$ as follows. We want to calculate the potential felt by an electron a distance $d$ from a finite periodic system of length $L$ and period $a$. We model our system by taking a length $L$ of an infinite line of electrons centred at $x$. This system is shown schematically in figure 5 .

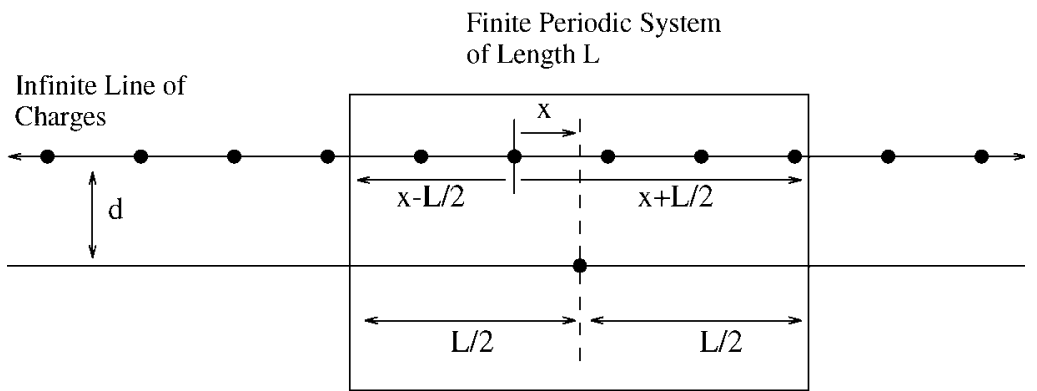

Figure 5. Schematic model of a periodic system of electrons. The potential on an electron centred at $x$ and a distance $d$ from an infinite line of electrons is obtained by taking a finite segment of length $L$ of the infinite chain centred at $x$.

This potential energy is given by

$$
V=\sum_{n=-\frac{1}{a}(L / 2-x)}^{\frac{1}{a}(L / 2+x)} \frac{e^{2}}{\sqrt{(x-n a)^{2}+d^{2}}}
$$


which can be rewritten as

$$
\begin{aligned}
V=\int_{-\frac{1}{a}(L / 2-x)}^{\frac{1}{a}(L / 2+x)} & \mathrm{d} z \frac{e^{2}}{\sqrt{(x-a z)^{2}+d^{2}}} \sum_{n=-\infty}^{\infty} \delta(z-n) \\
& =\int_{-\frac{1}{a}(L / 2-x)}^{\frac{1}{a}(L / 2+x)} \mathrm{d} z \frac{e^{2}}{\sqrt{(x-a)^{2}+d^{2}}} \sum_{l=-\infty}^{\infty} \mathrm{e}^{\mathrm{i} 2 \pi l z} \\
& =-\sum_{l=-\infty}^{\infty} \int_{-L / 2}^{L / 2} \frac{\mathrm{d} z^{\prime}}{a} \frac{e^{2}}{\sqrt{z^{\prime 2}+d^{2}}} \mathrm{e}^{\mathrm{i} 2 \pi \frac{l}{a}(x-z)} \\
& =\int_{-L / 2}^{L / 2} \frac{\mathrm{d} z^{\prime}}{a} \frac{e^{2}}{\sqrt{z^{\prime 2}+d^{2}}} \\
& +2 \sum_{l=1}^{\infty}\left[\int_{-L / 2}^{L / 2} \frac{\mathrm{d} z^{\prime}}{a} \frac{e^{2}}{\sqrt{z^{\prime 2}+d^{2}}} \cos \left(\frac{2 \pi l}{a} z^{\prime}\right)\right] \cos \left(\frac{2 \pi l}{a} x\right) .
\end{aligned}
$$

The first term is a constant in $x$, therefore we ignore it. For small separation distances $d / a \lesssim 1$, higher $l$ terms may be ignored and we keep only the $l=1$ term. This yields finally

$$
V \approx \frac{4}{a}\left[\int_{0}^{L / 2} \mathrm{~d} z^{\prime} \frac{\cos \left(\frac{2 \pi}{a} z^{\prime}\right)}{\sqrt{z^{\prime 2}+d^{2}}}\right] \cos \left(\frac{2 \pi}{a} x\right) .
$$

We can now identify the amplitude $V_{0}$ in equation (3) for a crystal with $N$ electrons as

$$
\begin{aligned}
V_{0} & =N \frac{4}{a} \int_{0}^{L / 2} \mathrm{~d} z^{\prime} \frac{\cos \frac{2 \pi}{a} z^{\prime}}{\sqrt{z^{\prime 2}+d^{2}}} \\
& =N \frac{4}{a} \int_{0}^{L / 2 a} \mathrm{~d} z \frac{\cos (2 \pi z)}{\sqrt{z^{2}+d^{2}}} .
\end{aligned}
$$

As mentioned in the introduction for a one-dimensional system, a long-range interaction will lead to the formation of a Wigner crystal [12, 13]. The long-range nature of the Coulomb force should be apparent for electron densities, $n \lesssim \frac{1}{10} a_{\mathrm{B}}$ where $a_{\mathrm{B}}$ is the Bohr radius for the particular ring material [21]. For a typical mesoscopic ring radius of $1 \mu \mathrm{m}$, crystal lattice constant $a=10 a_{\mathrm{B}}$ and $a_{\mathrm{B}} \approx 2 \AA$, we have $L / a>10^{3}$ so the upper limit in the integral in equation (8) may be taken to infinity, which yields

$$
V_{0}=\frac{4 N e^{2}}{a} K_{0}\left(2 \pi \frac{d}{a}\right)
$$

where $K_{0}$ is the zeroth-order Bessel function. This implies an exponential decrease in the amplitude of the interaction with increasing separation between the two Wigner crystals. Comparison with equation (7) shows that the two crystals will be 'locked' together at a distance $d / a \approx 4$. So two very low-density perfect Wigner crystals will be essentially locked together when separated by a distance of four times the lattice constant or less.

\subsection{Impurity effects}

We now consider the effects of impurities on the dynamics of the Wigner crystal. In the presence of an impurity, the phase of the Wigner crystal will be pinned. In this case the crystal ring cannot rotate as a whole so the current as presented in the last subsection is not possible. It was shown, however, by Rice et al [22], that a new type of current is possible, namely the tunnelling of solitons through the impurity barrier. It is the tunnelling of solitons that we will investigate in this section. 

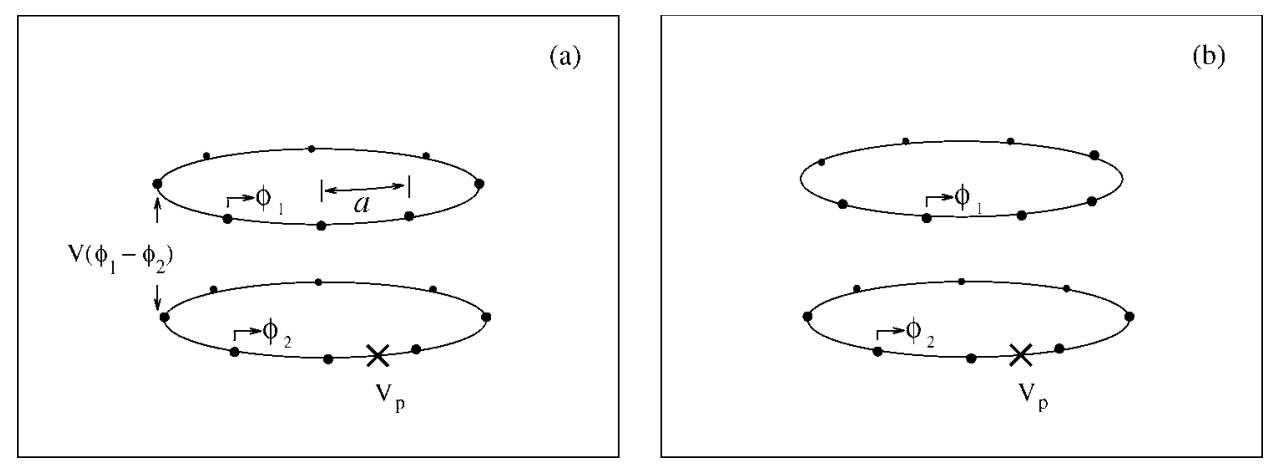

Figure 6. Schematic illustration of the two-ring Wigner crystal setup. The phase $\phi_{1,2}=$ $\frac{2 \pi}{a} u_{1,2}(x, t)$ where $u_{1,2}$ is the local displacement of the Wigner crystal. The interaction between the two rings is $V\left(\phi_{1}-\phi_{2}\right)=W\left[1-\cos \left(\phi_{1}-\phi_{2}\right)\right]$. Ring 2 has an impurity of strength $V_{p}$ that couples to the phase $\Phi_{2}$. Figure 6(b) shows a phase shift in the relative coordinate $\left(\phi_{1}-\phi_{2}\right)$ of $\pi$ relative to figure $6(\mathrm{a})$.

We are interested in knowing the ratio of the drag current in ring 2, to the drive current in ring 1 . We therefore take as an assumption the presence of a current in ring 1 and look for the relative magnitude of the drag current in ring 2 in the presence of a single impurity. This system is shown schematically in figure 6. Using equation (2), we take as a description of our two-ring Wigner crystal system, the following Lagrangian:

$$
\mathcal{L}=\mathcal{L}_{1}+\mathcal{L}_{2}-W\left[1-\cos \left(\phi_{1}-\phi_{2}\right)\right]
$$

where

$$
\begin{aligned}
& \mathcal{L}_{1}=\frac{m a}{8 \pi^{2}}\left\{{\frac{\partial \phi_{1}}{\partial t}}^{2}-s^{2}{\frac{\partial \phi_{1}}{\partial x}}^{2}\right\} \\
& \mathcal{L}_{2}=\frac{m a}{8 \pi^{2}}\left\{\left(\frac{\partial \phi_{2}}{\partial t}\right)^{2}-s^{2}\left(\frac{\partial \phi_{2}}{\partial x}\right)^{2}\right\}-V_{p} \delta(x)\left[1-\cos \left(\phi_{2}\right)\right]
\end{aligned}
$$

and the phase $\phi_{1,2}=u_{1,2}(x, t)(2 \pi / a)$.

The first two terms in equation (9) are the Lagrangians describing the dynamics of rings 1 and 2 respectively. The last term describes the interaction between the two rings. We follow the work of Krive et al [20] and introduce the dimensionless parameter

$$
\alpha=\frac{\pi \hbar}{m s a}
$$

which characterizes the magnitude of the quantum fluctuations of the Wigner crystal, and the parameter

$$
T_{s}=\hbar s
$$

which characterizes the energy scale in a Wigner crystal of length $L$. We will be concerned with stiff crystals that are weakly fluctuating, characterized by $\alpha \ll 1$.

The basic approach that we will use here is a semiclassical approximation used by Larkin and Lee [23] in the context of charge density waves and elaborated by Kleinert [24] for a single particle tunnelling through a barrier. In this approach the probability amplitude of a soliton tunnelling through an impurity is given by $\mathrm{e}^{A / \hbar}$ where $A$ is the action associated with the tunnelling trajectory obtained by minimizing the function $\int \mathrm{d} t \int \mathrm{d} x \mathcal{L}(\tau)$, where $\mathcal{L}(\tau)$ is 
the Lagrangian, equation (9), with $t$ replaced by the imaginary time $\tau=\mathrm{i} t$. This approach amounts to approximating the path integral of the propagator by its value at the classical trajectory [24].

We now want to answer the following question: given a current in ring 1 that causes a rotation of the phase by $2 \pi$, what is the probability that the phase of ring 2 will also rotate by $2 \pi$ ? It is apparent that there are three possibilities:

(i) $\phi_{1} \rightarrow 2 \pi, \phi_{2} \rightarrow 0$ : the phase in ring 2 will remain pinned and ring 1 will rotate on top of the relative potential between the two ending with a relative phase difference $\left(\phi_{1}-\phi_{2}\right)=2 \pi$

(ii) $\phi_{1} \rightarrow 2 \pi, \phi_{2} \rightarrow 2 \pi$ : the phase of ring 2 will tunnel through the impurity thus increasing by $2 \pi$ and ending in phase with $\phi_{1}$;

(iii) $\phi_{1} \rightarrow 2 \pi, \phi_{2} \rightarrow-2 \pi$ : the phase of ring 2 will counter-rotate with that in ring 1 ending with a relative phase difference, $\left(\phi_{1}-\phi_{2}\right)=4 \pi$.

Here we have limited our consideration to single tunnelling trajectories. In other words, each shift of the phase of each ring occurs due to a single tunnelling event and ignore all multistep tunnelling trajectories. Thus we only consider strong coupling between rings, $W L /\left(\frac{N \hbar^{2}}{m R^{2}}\right) \gtrsim 1$, and strong pinning $\alpha V_{p} \gg T_{s}$. We now calculate the tunnelling amplitudes for each case above.

In case (i), the Lagrangian in equation (9) reduces to

$$
\mathcal{L}=-\frac{m a}{8 \pi^{2}}\left\{\left(\frac{\partial \phi_{1}}{\partial \tau}\right)^{2}+s^{2}\left(\frac{\partial \phi_{1}}{\partial x}\right)^{2}\right\}-W\left[1-\cos \left(\phi_{1}\right)\right] .
$$

This is simply the problem of a single Wigner crystal ring in the presence of a periodic pinning potential. The classical wave equation resulting from this Lagrangian, in real time, is

$$
\frac{m a}{8 \pi^{2}}\left\{\ddot{\phi_{1}}-s^{2} \phi_{1}^{\prime \prime}\right\}+W \sin \left(\phi_{1}\right)=0 .
$$

This is the sine-Gordon equation and it admits the exact soliton solution

$$
\phi_{1}=4 \tan ^{-1}\left[\exp \pm\left(\frac{\tilde{x}-u \tilde{t}}{\sqrt{1-u^{2}}}\right)\right]
$$

where $\tilde{x}=\frac{\omega_{0}}{s} x, \tilde{t}=\omega_{0} t$ and

$$
\omega_{0}=\sqrt{\frac{4 \pi^{2} W}{m a}} .
$$

The + sign corresponds to a soliton rotating in the clockwise direction, the - sign corresponds to an antisoliton rotating in the counterclockwise direction and $u$ is the soliton/antisoliton velocity. The boundary conditions on such a solution are $\phi(-L / 2, t)=\phi(L / 2, t)$. A solution to this equation that matches this boundary condition is the soliton/antisoliton solution and has the form

$$
\phi_{1}(x, t)=4 \tan ^{-1}\left[\frac{u \sinh \left(\tilde{x} / \sqrt{1-u^{2}}\right)}{\cosh \left(u \tilde{t} / \sqrt{1-u^{2}}\right)}\right] .
$$

This solution has the following interpretation: at $t=0$ the phase of the crystal is 0 , as $t$ increases, the phase at $x=0$ locally increases to $2 \pi$ creating a region around $x=0$ with phase $2 \pi$. As $t$ increases this region with $2 \pi$ phase propagates symmetrically from $x=0$ at speed $u$ towards $x=-L / 2$ and $x=-L / 2$ until the entire ring is at $\phi=2 \pi$. The Lagrangian, equation (12), has been considered in the context of charge density wave tunnelling by Bogachek 
et al [25], and there it is noted that the homogeneous (space-independent) soliton solution is advantageous in the action against any spatially dependent solution for a finite system. The homogenous solution corresponds to a uniform rotation of the Wigner crystal as a whole and has the form [25]

$$
\phi_{1}(\tau)=4 \tan ^{-1} \exp \left(\omega_{0} \tau\right) .
$$

This solution describes the uniform increase of the phase of the entire ring from 0 to $2 \pi$ in a time $\tau_{0} \sim 1 / \omega_{0}$. The action associated with this solution is by equation (12)

$$
A_{1}=-\frac{8 W L}{\omega_{0}}=-8\left[\frac{W L}{\left(\frac{N}{m R^{2}}\right)}\right]^{1 / 2} \text {. }
$$

In case (ii), the relative difference between the two phases does not increase, while the phase of the centre of mass of the two-ring system increases by $2 \pi$. We assume that during the tunnelling process the two rings remain in phase to minimize the potential energy between them. If we rewrite equation (9) using $\phi=\phi_{1}-\phi_{2}$ and $\theta=\phi_{1}+\phi_{2}$, we get the relevant Lagrangian for case (ii) to be

$$
\mathcal{L}=-\frac{m a}{8 \pi^{2}}\left\{\left(\frac{\partial \theta}{\partial \tau}\right)^{2}+s^{2}\left(\frac{\partial \theta}{\partial x}\right)^{2}\right\}-V_{p} \delta(x)\left[1-\cos \left(\frac{\theta}{2}\right)\right] .
$$

This is the problem of a single Wigner crystal ring tunnelling through an impurity. We consider the case of strong crystal pinning, $\alpha V_{p} \gg T_{s}$.

The case of soliton tunnelling through an impurity in a strongly pinned charge density wave was first considered by Larkin and Lee [23]. In their analysis, the tunnelling process is broken into two stages. The first stage consists of a rapid tunnelling of a length $l_{0}$ of the crystal through the impurity in a short time $t_{0}$ and leaves the crystal in a distorted state of high potential energy. The second stage consists of a slow relaxation of the crystal from this distorted state back into a state that minimizes its potential energy.

To describe the first stage of the process Larkin and Lee proposed the following trial solution to equation (15):

$$
\theta=\frac{2 \pi t}{t_{0}}\left(1-\frac{|x|}{l_{0}}\right)
$$

for $0<t<t_{0}$ and $|x|>l_{0}$.

It is evident that this solution interpolates between the initial state, $\theta=0$ for all $x$ at $t=0$, and the final state $\theta=2 \pi$ for $x=0$ at $t=2 \pi$. The second stage of the process takes place away from the impurity $|x|>l_{0}$ and obeys the equation of motion

$$
\ddot{\theta}+s^{2} \theta^{\prime \prime}=0 .
$$

The solution to this stage of the tunnelling should obey the periodic boundary conditions of the ring and match the solution for the first stage at $x=-L / 2, L / 2$. This problem has been considered in the work of Krive et al [19] where the proposed trajectory for the relaxation stage has the form

$$
\phi=\pi \pm 2 \arctan \left(\frac{\left(\tau-\tau_{s}\right) s}{|x|}\right) .
$$

The action associated with the tunnelling stage of the process is

$$
A_{2 t}=-\frac{\hbar}{\alpha}\left(C_{1}+C_{2} \frac{\alpha V_{p}}{T_{h}}\right) \text {. }
$$


where $C_{1,2}$ are constants. The action associated with the relaxation stage is

$$
A_{2 r}=-\frac{\hbar}{\alpha} \ln \left(\frac{L}{2 l_{0}}\right)
$$

The length $l_{0}$ is obtained by minimizing the total action $A_{2 T}=A_{2 t}+A_{2 r}$ with the result that

$$
l_{0}=\frac{\hbar s}{C \alpha V_{p}}
$$

and

$$
A_{2 T}=-\frac{\hbar}{\alpha}\left\{\ln \left(\frac{\alpha V_{p}}{T_{s}}\right)+C_{3}\right\}
$$

where $C$ is a constant and $C_{3}$ is a constant assumed to be of order unity [23]. Equation (16) shows the interesting result that the action is only logarithmically dependent on the impurity for strong pinning.

In case (iii) above, the centre of mass of the two-ring system does not move while the phase of the relative coordinates increases by $4 \pi$. The relevant Lagrangian for this system is given by

$\mathcal{L}=-\frac{m a}{8 \pi^{2}}\left\{\left(\frac{\partial \phi}{\partial \tau}\right)^{2}+s^{2}\left(\frac{\partial \phi}{\partial x}\right)^{2}\right\}-V_{p} \delta(x)\left[1-\cos \left(\frac{\phi}{2}\right)\right]-W\left[1-\cos \left(\frac{\phi}{2}\right)\right]$

This is identical to the Lagrangian in equation (15) with the addition of the term proportional to $W$. Since the probability for this process goes as $\mathrm{e}^{A}$ it is expected that the probability for this case will be reduced relative to that in case (ii) by $\sim \mathrm{e}^{-W}$ and can therefore be neglected.

We are now in a position to analyse the relative magnitude of the drag current $J_{\mathrm{D}}$ to the drive current $J_{0}$. This ratio is given by

$$
\frac{J_{\mathrm{D}}}{J_{0}}=\frac{\mathrm{e}^{\frac{A_{2} T}{\hbar}}}{\mathrm{e}^{\frac{A_{1}}{\hbar}}+\mathrm{e}^{\frac{A_{2} T}{\hbar}}} .
$$

It is clear that if $\left|A_{1}\right| \gg\left|A_{2 T}\right|$ the two crystals will essentially be locked together. For purposes of illustration we consider a stiff crystals with $\alpha=0.1$ and consider values of $V_{p}$ for stiff pinning $\alpha V_{p} \gg T_{s}$.

Figure 7 shows that the impurity effects are not that drastic in determining the value of $W$ where the locking of the two crystals occur. This is due to the fact that the impurity potential enters logarithmically into the tunnelling action. Comparison with figure 4(b) shows that the distance at which the two Wigner crystals become 'locked' together is essentially the same as in the impurity-free case. The main effect of the impurity is then in the magnitude of the persistent current. 

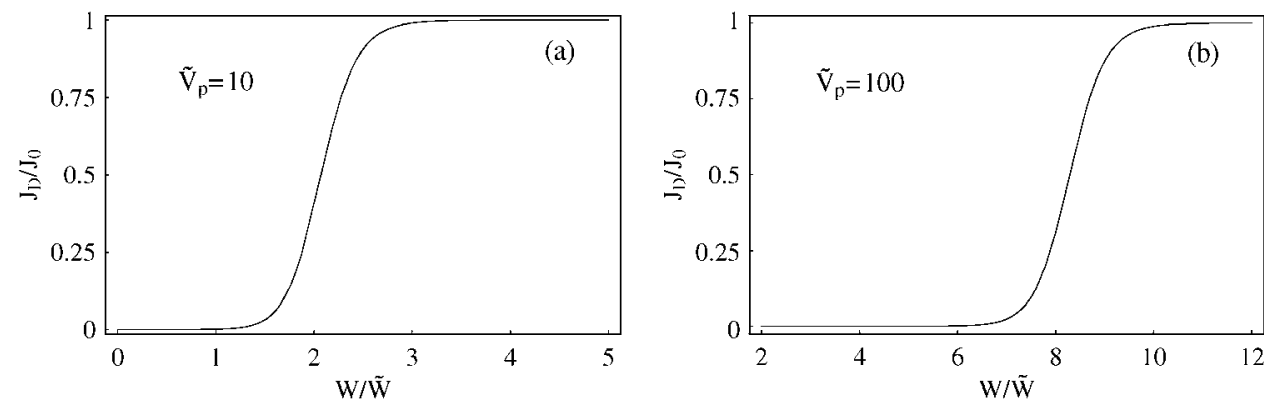

Figure 7. Plots $J_{\mathrm{D}} / J_{0}$ versus interaction strength $W$, for $\alpha=0.1$ and $\alpha V_{P} / T_{S}=10$ and 100 . The interaction strength is shown in units of $\tilde{W}=\frac{N \hbar}{m R^{2}}$ and $\tilde{V}_{p}=\frac{\alpha V_{p}}{T_{s}}$.

We can estimate the magnitude of these currents by noting that if two currents are locked together, then the magnitude of the persistent current for a given flux $\Phi$ should be the same as a single Wigner crystal ring with twice the mass. This current has been calculated at zero temperature to be [19]

$$
J_{0} \sim T_{s}\left(\frac{T_{s}}{\alpha V_{p}}\right)^{1 / \alpha} \mathrm{e}^{-C_{3} / \alpha} \cos \left(2 \pi \frac{\Phi}{\Phi_{0}}\right) .
$$

We see that the current in equation (19) is much smaller than the disorder-free persistent current due to the $\left(1 / V_{p}\right)^{1 / \alpha}$ factor.

In summary, we have analysed the Coulomb drag between two one-dimensional Wigner crystal rings. For sufficiently large interaction between rings the drag current is essentially equal in magnitude to the drive current. For an impurity free ring this 'locking' of currents occurs at a separation distance of $d \approx 4 a$ with $a$ the crystal period. We analysed the effect of a single impurity in the drag ring and found that the two currents are 'locked' at essentially the same distance and the major effect of the impurity is to significantly decrease the magnitude of the persistent current.

\section{References}

[1] Lilly M P, Eisenstein J P, Pfeiffer L N and West K W 1998 Phys. Rev. Lett. 801714

[2] Gramila T J, Eisenstein J P, MacDonald A H, Pfeiffer L N and West K W 1991 Phys. Rev. Lett. 661216

[3] Rojo A G 1999 J. Phys.: Condens. Mattter 11 R31

[4] Zheng L and MacDonald A H 1993 Phys. Rev. B 488203

[5] Pogrebrinskiǐ M B 1977 Fiz. Tekh. Poluprovodn. 116371977 Sov. Phys. Semicond. (Engl. Trans.) 11372

[6] Price P J 1983 Physica B + C 117/118 750

[7] Gurevich V L, Pevzner V B and Fenton E W 1997 J. Phys.: Condens. Matter 101551

[8] Raichev O E and Vasilopoulos P 1999 Phys. Rev. Lett. 831551

[9] Raichev O and Vasilopoulos P 2000 Phys. Rev. B 617511

[10] Gurevich V L and Muradov M I 2000 JETP Lett. 71111

[11] Debray P, Vasilopoulos P, Raichev O, Perrin R, Rahman M and Mitchel W C 2000 Physica E 6694

[12] Schulz H J 1993 Phys. Rev. Lett. 711864

[13] Fano G, Ortolani F, Parola A and Ziosi L 1999 Phys. Rev. B 6015654

[14] Doering C 1999 Private communication

[15] Cladis P E, Parks R D and Daniels J M 1968 Phys. Rev. Lett. 211521

[16] Rojo A G and Mahan G D 1992 Phys. Rev. Lett. 682074

[17] Baker J, Vignale G and Rojo A G 1999 Phys. Rev. B 608804

[18] Glazman L I, Ruzin I M and Shklovskii B I 1992 Phys. Rev. Lett. 458454

[19] Krive I V, Sandström P, Shekter R I, Girvin S M and Jonson M 1995 Phys. Rev. B 5216451 
[20] Jordan D W and Smith P 1977 Nonlinear Ordinary Differential Equations: an Introduction to Dynamical Systems (New York: Academic)

[21] Ivanov A L and Haug H 1993 Phys. Rev. Lett. 713182

[22] Rice M J, Bishop A R, Krumhansl J A and Trullinger S E 1976 Phys. Rev. Lett. 36432

[23] Larkin A I and Lee P A 1978 Phys. Rev. B 171596

[24] Kleinert H 1995 Path Integrals in Quantum Mechanics, Statistics and Polymer Physics (Singapore: World Scientific)

[25] Bogachek E N, Krive I V, Kulik I O and Rozhavsky A S 1990 Phys. Rev. B 427614 